PROCEEDINGS OF THE

AMERICAN MATHEMATICAL SOCIETY

Volume 125, Number 4, April 1997, Pages 1063-1070

S 0002-9939(97)03864-1

\title{
DUAL LOCAL COMPLETENESS
}

\author{
STEPHEN A. SAXON AND L. M. SÁNCHEZ RUIZ \\ (Communicated by Dale Alspach)
}

\begin{abstract}
The 1971 articles in which Saxon-Levin and Valdivia independently proved their Theorem feature two conditions equivalent to dual local completeness. One became Ruess' property (LC). The other is among new characterizations previously known only as necessary conditions.
\end{abstract}

\section{INTRODUCTION}

A sequence $\sigma=\left\{A_{n}: n \in \mathbb{N}\right\}$ of absolutely convex subsets of a locally convex space $E(\mathcal{T})$ is absorbing in $E$ if it is increasing and each $x \in E$ is absorbed by some $A_{n}$; then we denote by $\mathcal{T}_{\sigma}$ the finest locally convex topology on $E$ that induces the same topology as $\mathcal{T}$ on each $A_{n}$. (Cf. [1], [8].) The topology $\mathcal{T}_{\sigma}$ is defined by the family of those seminorms whose restrictions to the sets $A_{n}$ are continuous for the topology induced on $A_{n}$ by $\mathcal{T}$. In Valdivia's development of the Saxon-LevinValdivia Theorem, he showed that $\mathcal{T}=\mathcal{T}_{\sigma}$ always holds when $E(\mathcal{T})$ is barrelled ([16], Theorem 5). Ruess [9] defined a space $E(\mathcal{T})$ to have property $(L)$ if $\mathcal{T}=\mathcal{T}_{\sigma}$ holds for each absorbing sequence $\sigma$, and to have the weaker property $(L C)$ if each $\mathcal{T}_{\sigma}$ is compatible with the dual pair $\left(E, E^{\prime}\right)$. Thus

$$
\text { barrelled } \Rightarrow \text { property (L) } \Rightarrow \text { property (LC). }
$$

Furthermore, every barrelled space has its Mackey topology, and for any Mackey space $E$, it is clearly true that $E$ has property (L) if and only if $E$ has property (LC). Valdivia explicitly pointed out that [ barrelled $\Rightarrow$ property (LC)], and the latter property, according to Ruess (see below), is equivalent to dual local completeness.

The crux of the Saxon-Levin proof was to show that every barrelled space $E$ has the property that any countable-codimensional subspace spanned by a closed absolutely convex subset of $E$ is, itself, closed ([15], §3, Lemma). We will show this property, also, is equivalent to dual local completeness, which thus provides an unexpected common footing for both papers. This and other new equivalences unify vital ideas already in book form (e.g., [5], [6], [7], [19], [22]).

Every space will be assumed a Hausdorff locally convex space over the scalar field of real or complex numbers; "countable" means "finite or denumerable".

Received by the editors September 15, 1995.

1991 Mathematics Subject Classification. Primary 46A08.

Key words and phrases. Dual locally complete, barrelled, properties (L) and (LC).

This paper was started while the second author stayed at the University of Florida supported by DGICYT PR95-182, later by PR94-204 and IVEI 003/033.

(C) 1997 American Mathematical Society 


\section{DuAl LOCAL COMPLETENESS}

When $\sigma=\{A\}$, we will denote $\mathcal{T}_{\sigma}$ as $\mathcal{T}_{A}$. Ruess ([11], Propositions 2.2, 2.3) asserts without proof that a space $E(\mathcal{T})$ has property $(\mathrm{L})$ (respectively, $(\mathrm{LC})$ ) if and only if, given any absorbing absolutely convex set $A$ (respectively, any $f \in E^{*}$ such that $\left.f\right|_{A}$ is continuous), then $\mathcal{T}=\mathcal{T}_{A}$ (respectively, $f \in E^{\prime}$ ). We prove Ruess' characterizations with new ones involving $\ell^{1}$.

Recall that a space $E$ is locally complete if and only if every bounded closed absolutely convex subset of $E$ is a Banach disk [7]. $E$ is $\ell^{1}$-complete if, for each $\left\{\lambda_{n}\right\}_{n} \in \ell^{1}$ and bounded sequence $\left\{x_{n}\right\}_{n} \subset E$, the series $\sum_{n} \lambda_{n} x_{n}$ converges in $E$.

Theorem 2.1. A space $E$ is locally complete if and only if it is $\ell^{1}$-complete.

Proof. Suppose $E$ is locally complete and we are given $\left\{\lambda_{n}\right\}_{n} \in \ell^{1}$ and a bounded sequence $\left\{x_{n}\right\}_{n}$ in $E$. The closed absolutely convex hull $A$ of $\left\{x_{n}\right\}_{n}$ is a Banach disk. Now $q>p$ implies

$$
\sum_{n \leq q} \lambda_{n} x_{n}-\sum_{n<p} \lambda_{n} x_{n} \in\left(\left|\lambda_{p}\right|+\ldots+\left|\lambda_{q}\right|\right) A .
$$

Thus $\sum_{n} \lambda_{n} x_{n}$ converges in the Banach space $E_{A}$, and so must also converge in the relatively coarser topology of $E$.

Conversely, suppose $E$ is $\ell^{1}$-complete and $A$ is a closed disk in $E$, with $\left\{y_{n}\right\}_{n}$ a Cauchy sequence in the normed space $E_{A}$. There is an increasing sequence $\left\{n_{k}\right\}_{k} \subset$ $\mathbb{N}$ such that $\left\|y_{q}-y_{p}\right\| \leq 2^{-k}$ whenever $p, q, k \in \mathbb{N}$ with $p, q \geq n_{k}$. If $\lambda_{k}=2^{-k}$ and $x_{k}=2^{k}\left(y_{n_{k+1}}-y_{n_{k}}\right)$, then $\left\{x_{k}\right\}_{k} \subset A$ is bounded and $\sum_{n} \lambda_{n} x_{n}$ converges to some $x$ in $E$. Given $j>k$, we have

$$
\sum_{n \leq j} \lambda_{n} x_{n}-\sum_{n<k} \lambda_{n} x_{n} \in\left(2^{-k}+\ldots+2^{-j}\right) A \subset 2^{-k+1} A .
$$

For $k$ fixed, $2^{-k+1} A$ is closed in $E$, and

$$
x-\sum_{n<k} \lambda_{n} x_{n}=\lim _{j}\left(\sum_{n \leq j} \lambda_{n} x_{n}-\sum_{n<k} \lambda_{n} x_{n}\right) \in 2^{-k+1} A .
$$

Therefore as $k$ increases, $y_{n_{k}}=y_{n_{1}}+\sum_{n<k} \lambda_{n} x_{n}$ tends to $y_{n_{1}}+x$ in $E_{A}$, as does, then, $\left\{y_{n}\right\}_{n}$. We conclude that $A$ is a Banach disk and $E$ is locally complete.

Obviously, every sequentially complete space is locally complete (cf. [7]).

Theorem 2.2. Given a space $E(\mathcal{T})$, the following assertions are equivalent:

(1) (Ruess) E has property (L).

(2) (Ruess) $\mathcal{T}=\mathcal{T}_{A}$ for any absorbing absolutely convex subset $A$ of $E$.

(3) If $\left\{\lambda_{n}\right\}_{n} \in \ell^{1}$ and $\left\{p_{n}: n \in \mathbb{N}\right\}$ is a pointwise bounded sequence of continuous seminorms on $E$, then $\sum_{n}\left|\lambda_{n}\right| p_{n}$, pointwisely defined, is a continuous seminorm on E.

Proof. $[(1) \Rightarrow(2)]$ is clear.

$[(2) \Rightarrow(3)]$. Let $A=\bigcap_{n}\left\{x \in E: p_{n}(x) \leq 1\right\}$. To see that $p=\sum_{n}\left|\lambda_{n}\right| p_{n}$ is continuous, we need only see that $\left.p\right|_{A}$ is continuous because of (2), and for this we just check that $\left.p\right|_{A}$ is continuous at the origin (Garling; cf. [9], Lemma $3.3(1))$. Given $\epsilon>0$, choose $k$ such that $\sum_{n>k}\left|\lambda_{n}\right|<\epsilon / 2$. Continuity supplies a 0-neighborhood $W$ in $E$ with $\sum_{n \leq k}\left|\lambda_{n}\right| p_{n}(x)<\epsilon / 2$ for $x \in W$. Hence for each 
$x \in W \cap A$ we have $p(x) \leq \sum_{n \leq k}\left|\lambda_{n}\right| p_{n}(x)+\sum_{n>k}\left|\lambda_{n}\right|<\epsilon / 2+\epsilon / 2=\epsilon$; i.e., $\left.p\right|_{A}$ is continuous at the origin.

$[(3) \Rightarrow(1)]$. Let $\sigma=\left\{A_{n}: n \in \mathbb{N}\right\}$ be an absorbing sequence of $E(\mathcal{T})$; then $\sigma^{*}=\left\{2^{n} A_{n}: n \in \mathbb{N}\right\}$ is an absorbing sequence of $E$, also, and $\mathcal{T}_{\sigma}=\mathcal{T}_{\sigma^{*}}$. A base of 0 -neighborhoods for $\mathcal{T}_{\sigma^{*}}$ is given by all the sets $W=U_{0} \cap\left(\bigcap\left\{\overline{2^{n} A_{n}+U_{n}}: n \in \mathbb{N}\right\}\right)$, where $\left\{U_{n}: n=0,1,2, \ldots\right\}$ runs through all the sequences of absolutely convex neighborhoods of the origin in $E(\mathcal{T})$. (See Lemma 1 of [8], or 8.1.12 of [7].) We need show that any such $W$ is a $\mathcal{T}$-neighborhood of the origin. If $p_{n}$ is the gauge of $2^{n} A_{n}+$ $U_{n}$, then $\left\{2^{n} p_{n}: n \in \mathbb{N}\right\}$ is a family of continuous seminorms defined on $E$, and is pointwise bounded since $\sigma$ is absorbing. By (3), we have $p=\sum_{n=1}^{\infty} \frac{1}{2^{n}} 2^{n} p_{n}$ is a continuous seminorm on $E$. Therefore $p^{-1}([0,1])$ is a $\mathcal{T}$-neighborhood contained in $\bigcap\left\{p_{n}^{-1}([0,1]): n \in \mathbb{N}\right\} \subset \bigcap\left\{\overline{2^{n} A_{n}+U_{n}}: n \in \mathbb{N}\right\}$, so that $W$ is a $\mathcal{T}$-neighborhood of the origin.

Recall that a space $E$ is dual locally complete [17] if $E^{\prime}\left(\sigma\left(E^{\prime}, E\right)\right)$ is locally complete. $E$ is dual $\ell^{1}$-complete if $E^{\prime}\left(\sigma\left(E^{\prime}, E\right)\right)$ is $\ell^{1}$-complete.

Ruess' Final Remarks [10] imply that ([7], 8.1.29(i)) dual local completeness is equivalent to property (LC). And $[E$ is barrelled $] \Rightarrow\left[E\right.$ has property (S); i.e., $E^{\prime}$ is $\sigma\left(E^{\prime}, E\right)$-sequentially complete $] \Rightarrow\left[E\right.$ is dual $\ell^{1}$-complete $]$. De Wilde proved that every metrizable space with property $(S)$ is barrelled. Saxon's proof ([13], Theorem 2.7 ) actually used dual $\ell^{1}$-completeness instead of property (S).

Theorem 2.3. For any space $E(\mathcal{T})$ the following assertions are equivalent:

(1) (Ruess) $E$ is dual locally complete.

(2) $E$ is dual $\ell^{1}$-complete.

(3) (Ruess) E has property (LC).

(4) (Ruess) If $A$ is an absorbing absolutely convex set and $f \in E^{*}$ with $\left.f\right|_{A}$ continuous, then $f \in E^{\prime}$.

Proof. $[(1) \Leftrightarrow(2)]$. By Theorem 2.1 .

$[(2) \Rightarrow(3)]$. Let $\left\{B_{n}: n \in \mathbb{N}\right\}$ be an increasing sequence of closed absolutely convex sets covering $E$ (cf. 8.1.17(i) of [7]) and suppose that $f \in E^{*}$ has a continuous restriction on each $B_{n}$. We must show that $f \in E^{\prime}$. If $f=0$, this is trivial; otherwise, choose $x \in E$ such that $f(x)=1$. Then, for each $n \in \mathbb{N}$, we have $C_{n}=B_{n} \cap f^{-1}(\{0\})$ is closed and absolutely convex and misses $2^{-n} x$; the bipolar theorem yields $f_{n} \in E^{\prime}$ with $f_{n}(x)=1$ and $\left|f_{n}(y)\right|<2^{-n}$ for every $y \in C_{n}$. Since $\left\{B_{n}: n \in \mathbb{N}\right\}$ increases and covers $E$, the sequence $\left\{2^{n}\left(f_{n+1}-f_{n}\right): n \in \mathbb{N}\right\}$ is $\sigma\left(E^{\prime}, E\right)$-bounded, so that by (2) we have $f=f_{1}+\sum_{n} 2^{-n}\left[2^{n}\left(f_{n+1}-f_{n}\right)\right]$ is in $E^{\prime}$.

$[(3) \Rightarrow(4)]$. The sequence $\left\{A_{n}=A\right\}_{n}$ is absorbing since $A$ is.

$[(4) \Rightarrow(2)]$. Given $\left\{\lambda_{n}\right\}_{n} \in \ell^{1}$ and a $\sigma\left(E^{\prime}, E\right)$-bounded sequence $\left\{f_{n}\right\}_{n}$, let $f \in E^{*}$ be the pointwise limit of $\sum_{n} \lambda_{n} f_{n}$. To show that $f \in E^{\prime}$, it suffices to show that the restriction of $f$ to $A=\left\{f_{n}: n \in \mathbb{N}\right\}^{\circ}$ is continuous at the origin, using (4). For $\epsilon>0$, choose $k$ such that $\sum_{n>k}\left|\lambda_{n}\right|<\epsilon / 2$, and choose a 0-neighborhood $U$ in $E$ such that $\left|\left(\sum_{n \leq k} \lambda_{n} f_{n}\right)(x)\right|<\epsilon / 2$ for all $x \in U$. Thus $x \in U \cap A$ implies $|f(x)| \leq\left|\left(\sum_{n \leq k} \lambda_{n} f_{n}\right)(x)\right|+\sum_{n>k}\left|\lambda_{n}\right|<\epsilon$, as required.

Let us mention a property weaker than dual locally complete. Valdivia showed ([16], Theorem 6 ) that in a barrelled space, if each member of an absorbing sequence 
$\left\{A_{n}: n \in \mathbb{N}\right\}$ is closed, then any bounded set is absorbed by some $A_{n}$. This was extended in ([1], Corollary 1) to $\ell^{\infty}$-barrelled spaces, further in ([9], Lemma 6.3) to spaces with property (LC). Ruess' [10], Lemma 1.2 justifies substituting the italicized statement for the definition of his property $(B)$. On the other hand, a space $E$ has the Banach-Mackey property if every $\sigma\left(E^{\prime}, E\right)$-bounded subset of $E^{\prime}$ is $\beta\left(E^{\prime}, E\right)$-bounded (cf. [2], p.3). Webb ([20], Proposition 4.1(1)) proved that every $c_{0}$-barrelled space has the Banach-Mackey property $\left(c_{0}\right.$-barrelled $\Rightarrow$ dual locally complete [7], 8.2.23(b)), and Pérez Carreras/Bonet [7], 5.1.34, states that every barrel in a dual locally complete space is bornivorous. All are corollaries to the Banach-Mackey Theorem, as we now show. $([(2) \Leftrightarrow(3)]$ is $[22]$, Theorem 10-4-7.)

Theorem 2.4. The following assertions about a space $E$ are equivalent:

(1) E has property (B).

(2) (Wilansky) Each barrel $U$ of $E$ is bornivorous.

(3) (Wilansky) E has the Banach-Mackey property.

Proof. $[(1) \Rightarrow(2)]$. Obvious.

$[(2) \Rightarrow(3)]$. Let $C$ be a $\sigma\left(E^{\prime}, E\right)$-bounded subset of $E^{\prime}$ and let $B$ be a bounded subset of $E$. By (2), there is some $\epsilon>0$ such that $\epsilon B$ is contained in the barrel $C^{\circ}$. Thus $C \subset C^{\circ \circ} \subset(\epsilon B)^{\circ}=1 / \epsilon\left(B^{\circ}\right)$, so that $B^{\circ}$ absorbs $C$; i.e., $C$ is $\beta\left(E^{\prime}, E\right)$ bounded.

$[(3) \Rightarrow(1)]$. Let $\left\{A_{n}: n \in \mathbb{N}\right\}$ be an absorbing sequence of closed subsets of $E$ and let $B$ be a bounded subset of $E$. Suppose $(1 / n) B \nsubseteq A_{n}$ for all $n$. The bipolar theorem provides $x_{n} \in B$ and $f_{n} \in A_{n}^{\circ}$ such that $\left|f_{n}\left(x_{n}\right)\right|>n$. Because $\left\{A_{n}: n \in \mathbb{N}\right\}$ is absorbing, $\left\{f_{n}: n \in \mathbb{N}\right\}$ is $\sigma\left(E^{\prime}, E\right)$-bounded, but not $\beta\left(E^{\prime}, E\right)$ bounded since it is not absorbed by $B^{\circ}\left(\left\{x_{n}: n \in \mathbb{N}\right\} \subset B\right)$, contradicting (3).

Theorem 2.5 (Banach-Mackey). Dual locally complete spaces have the BanachMackey property.

Proof. Let $C$ be a $\sigma\left(E^{\prime}, E\right)$-bounded subset of $E^{\prime}$ with $E_{C^{\circ \circ}}^{\prime}$ a Banach space, and let $B$ be a bounded subset of $E$. The Banach-Steinhaus Theorem assures that $B$, considered as a pointwise bounded subset of the dual of $E_{C^{\circ 0}}^{\prime}$, is uniformly bounded on the unit ball $C^{\circ \circ}$, thus on $C$, and so $B^{\circ}$ absorbs $C$.

Note. This is argument a) in Köthe's book ([5], §20.11.3). The alternate argument b) implicitly uses [dual locally complete $\Rightarrow$ dual $\ell^{1}$-complete]. The terminology "dual locally complete" [17] is subsequent to Köthe's book; we have modernized the statement of the Banach-Mackey Theorem, not its proof. Trivially, every quasibarrelled space with the Banach-Mackey property is barrelled, and thus every metrizable dual locally complete space is barrelled, an important weak barrelledness fact (re-)proved by Ruess ([9], Proposition 6.8).

Wilansky [22] defined a Mazur space to be one in which every sequentially continuous linear form is continuous.

Theorem 2.6. A Mazur space $E$ is dual locally complete if (and only if) it has the Banach-Mackey property.

Proof. We will show that $E$ is dual $\ell^{1}$-complete. Let $\left(\lambda_{n}\right)_{n} \in \ell^{1}$ and $\left\{f_{n}: n \in \mathbb{N}\right\}$ a $\sigma\left(E^{\prime}, E\right)$-bounded sequence be given, and set $f(x)=\sum_{n} \lambda_{n} f_{n}(x)$ for each $x \in E$. To show $f \in E^{\prime}$, we need only show $\lim _{n} f\left(x_{n}\right)=0$ for $\left\{x_{n}\right\}$ an arbitrary null sequence in $E$. The Banach-Mackey property provides $M>0$ such that $\left|f_{n}\left(x_{j}\right)\right| \leq$ 
$M$ for all $n, j$. Given $\epsilon>0$, choose $P \geq 1$ such that $\sum_{n>P}\left|\lambda_{n}\right|<\epsilon /(2 M)$. Continuity yields $Q$ such that $j>Q$ implies $\left|\sum_{n \leq P} \lambda_{n} f_{n}\left(x_{j}\right)\right|<\epsilon / 2$. Thus $j>Q$ implies

$$
\left|f\left(x_{j}\right)\right| \leq\left|\sum_{n \leq P} \lambda_{n} f_{n}\left(x_{j}\right)\right|+\sum_{n>P}\left|\lambda_{n}\right| M<\epsilon / 2+M \epsilon /(2 M)=\epsilon
$$

as desired.

A space $E$ is $c_{0}$-barrelled if each $\sigma\left(E^{\prime}, E\right)$-null sequence is equicontinuous [7].

Theorem 2.7. Each $c_{0}$-barrelled Mazur space E has property (S).

Proof. Suppose $f \in E^{*}$ satisfies $f(x)=\lim _{n} f_{n}(x) \quad(x \in E)$ for some sequence $\left\{f_{n}\right\}_{n} \subset E^{\prime}$. Assume $f$ is not continuous, hence not sequentially continuous, so that there exists a null sequence $\left\{x_{n}\right\}_{n}$ in $E$ for which $\left\{f\left(x_{n}\right)\right\}_{n}$ does not converge to the scalar 0 . Thus there is a null sequence $\left\{y_{k}\right\}_{k}$ in $E$ of the form $\left\{y_{k}\right\}_{k}=$ $\left\{x_{n_{k}} / f\left(x_{n_{k}}\right)\right\}_{k}$. Now each $f\left(y_{k}\right)=1$. For each $n$, continuity of $f_{n}$ yields $k_{n} \geq n$ such that $\left|f_{n}\left(y_{k_{n}}\right)\right| \leq 1 / 4$; choose $p_{n} \geq n$ such that $\left|f_{p_{n}}\left(y_{k_{n}}\right)\right| \geq 3 / 4$. Since $\left\{f_{n}\right\}_{n}$ is $\sigma\left(E^{\prime}, E\right)$-Cauchy, $\left\{f_{n}-f_{p_{n}}\right\}_{n}$ is $\sigma\left(E^{\prime}, E\right)$-null, and thus equicontinuous. Let $V$ be a 0 -neighborhood in $E$ with $\left|\left(f_{n}-f_{p_{n}}\right)(y)\right|<1 / 2$ for all $n$ and all $y \in V$. But for all $y_{k_{n}} \in V$ we have

$$
\left|\left(f_{n}-f_{p_{n}}\right)\left(y_{k_{n}}\right)\right| \geq\left|f_{p_{n}}\left(y_{k_{n}}\right)\right|-\left|f_{n}\left(y_{k_{n}}\right)\right| \geq 3 / 4-1 / 4=1 / 2,
$$

a contradiction.

A Mackey space is dual locally complete if and only if it is $c_{0}$-barrelled ([7], 5.1.33), so the two results above have the following corollary; equivalence of the last three statements is due to Kalton [4].

Corollary 2.8. For a Mackey Mazur space $E$ the following assertions are equivalent:

(1) $E$ is dual locally complete.

(2) (Kalton) E has the Banach-Mackey property.

(3) (Kalton) $E$ has property (S).

(4) (Kalton) $E$ is $c_{0}$-barrelled.

New characterizations of dual local completeness influenced by Saxon-Levin conclude the section. In the preliminary result, we more easily write $A=A^{\circ \circ}$ to indicate that $A$ is absolutely convex and closed.

Proposition 2.9. If $A=A^{\circ \circ} \subset E$ and $H$ is any finite-dimensional subspace of $E$, then $A+H=(A+H)^{\circ \circ}$.

Proof. By finite induction, it suffices to consider $H$ the span of a single element $x \in E$. Trivially, $A+H$ is absolutely convex; we must show the set is closed. Two cases may occur:

(i) $H \subset A$. Then for any $u \in A$ and $v \in H$, each $n v \in A$, so that we have

$$
u+v=\lim _{n}[(1-1 / n) u+1 / n(n v)] \in A
$$

since $A$ is convex and closed. That is, $A+H \subset A$. Hence $A+H=A$ is closed. 
(ii) There exists some $z \in H \backslash A$. Then the bipolar theorem yields $h \in A^{\circ}$ such that $h(z)>1$. If $t \in \overline{A+H}$, then $t \in \overline{\left(t+\{h\}^{\circ}\right) \cap(A+H)}$ since $t+\{h\}^{\circ}$ is a neighborhood of $t$. It suffices to prove $\left(t+\{h\}^{\circ}\right) \cap(A+H) \subset$ $A+\{\lambda z:|\lambda| \leq|h(t)|+2\}$, since the latter is a closed subset of $A+H$. Suppose $s \in\left(t+\{h\}^{\circ}\right) \cap(A+H)$. Then $|h(s)| \leq|h(t)|+1$ and $s=a+\lambda z$ for some $a \in$ $A$ and some scalar $\lambda$. [H is spanned by $z$. $]$ Hence $|h(s)|=|h(a)+\lambda h(z)| \geq$ $|\lambda| h(z)-1 \geq|\lambda|-1$. We conclude that $|\lambda| \leq|h(t)|+2$.

Theorem 2.10. In addition to (1)-(4) of Theorem 2.3, dual locally completeness of a space $E(\mathcal{T})$ is also equivalent to each of the following statements:

(5) If $F=\bigcup_{n} A_{n}$ is a countable-codimensional subspace of $E$ with each $A_{n}=$ $A_{n}^{\circ \circ} \subset A_{n+1}$, then $F$ is closed.

(6) If $f \in E^{*}$ and $\operatorname{sp}(A)$ is a countable-codimensional subspace of $E$ with $A=A^{\circ \circ}$ such that $f(A)=\{0\}$, then $f \in E^{\prime}$.

(7) If $F$ and $H$ are transverse subspaces of $E$ with $F=s p(A), A=A^{\circ \circ}$ and $\operatorname{dim}(E / F) \leq \aleph_{0}$, then both $F+H$ and $A+H$ are closed.

(8) If $F=\operatorname{sp}(A) \subset E$ with $A=A^{\circ \circ}$ and $\operatorname{dim}(E / F) \leq \aleph_{0}$, then $F$ is closed.

Proof. $[(3) \Rightarrow(5)]$. Suppose $x \in E \backslash F$ and let $\left\{x_{n}: n \in \mathbb{N}\right\}$ span an algebraic complement of $F+s p(\{x\})$ in $E$. Then $B_{n}=A_{n}+\{\lambda x:|\lambda| \leq 1\}+s p\left(\left\{x_{1}, \ldots, x_{n}\right\}\right)$ defines an absorbing sequence $\left\{B_{n}\right\}_{n}$ in $E$. Let us define $f \in E^{*}$ such that $f\left(F+s p\left(\left\{x_{n}: n \in \mathbb{N}\right\}\right)\right)=\{0\}$ and $f(x)=1$. Given any closed subset $S$ of the scalar field and $n \in \mathbb{N}$, we have

$$
f^{-1}(S) \cap B_{n}=A_{n}+\operatorname{sp}\left(\left\{x_{1} \ldots, x_{n}\right\}\right)+\{\lambda x:|\lambda| \leq 1 \text { and } \lambda \in S\}
$$

is closed via Proposition 2.9, so that $\left.f\right|_{B_{n}}$ is continuous. Therefore $f \in E^{\prime}, x \notin \bar{F}$, and $F$ is closed.

$[(5) \Rightarrow(6)]$. Let $\left\{H_{n}: n \in \mathbb{N}\right\}$ be an increasing sequence of finite-dimensional subspaces of $E$ whose union is an algebraic complement of $s p(A)$ in $f^{-1}(\{0\})$. Then $f^{-1}(\{0\})=\bigcup\left\{n A+H_{n}: n \in \mathbb{N}\right\}$ is closed by (5), so that $f \in E^{\prime}$.

$[(6) \Rightarrow(7)] . F+H$ is closed since, by (6), every linear form vanishing on $F+H$ is continuous. To see that $A+H$ is closed, we suppose $x \in(F+H) \backslash(A+H)$ and prove $x \notin(A+H)^{\circ \circ}$. Now $x=u+v$ with $u \in F \backslash A$ and $v \in H$. Since $u \notin A^{\circ \circ}$, there exists $g \in A^{\circ}$ with $g(u)>1$. Choose $h \in E^{*}$ with $h(F)=\{0\}$ and $\left.h\right|_{H}=\left.g\right|_{H}$. By (6), $h$ is continuous, and so must $f=g-h$ be, also. Hence $\left.f\right|_{F}=\left.g\right|_{F}$ and $f(H)=\{0\}$ yield $f \in(A+H)^{\circ}$, while $f(x)=g(u)>1$; i.e., $x \notin(A+H)^{\circ \circ}$.

$[(7) \Rightarrow(8)]$. Take $H=\{0\}$.

$[(8) \Rightarrow(4)]$. Let $C$ be an absolutely convex absorbing set in $E$, and suppose $f \in E^{*}$ with $\left.f\right|_{C}$ continuous. We must show $f \in E^{\prime}$. If $B=\bar{C}$, then $\left.f\right|_{B}$ is also continuous ([7], 8.1.17(i)). Thus $A=B \cap f^{-1}(\{0\})$ is closed, and so is $f^{-1}(\{0\})=s p(A)$, from $(8)$.

Note that Ruess' Proposition 3.5(1) [10] just says (5) is a necessary condition for property (LC), and Saxon-Levin (cf. [15], [21] or 8.2.17 of [7]) gives condition (8) as necessary for property $(\mathrm{S})$. 


\section{Permanence properties, Remarks}

Consolidation of dual local completeness affords simple proofs of some results in the Valdivia [16] and Saxon-Levin [15] papers: Every barrelled space is dual $\ell^{1}$ complete; Theorem 2.3 gives property (LC) which, in the presence of the Mackey topology, is property (L), yielding Valdivia's Theorem 5 and Corollary 2.5 in [16]. On the other hand, Saxon-Levin developed condition (8) of Theorem 2.10, which immediately solves their dense countable-codimensional subspace case. (They had solved closed subspaces by early 1968 and had the full result later that year [14]; Saxon gave a proof in his 1969 dissertation [12].)

Another immediate consequence of $[(1) \Leftrightarrow(8)]$ is Theorem 13 in [18]:

Theorem 3.1 (Valdivia). Every countable-codimensional subspace $F$ of a dual locally complete space $E$ is dual locally complete.

Proof. If $A$ is an absolutely convex closed subset of $F$ that spans a countablecodimensional subspace of $F$, its closure $\bar{A}$ plays a similar role in $E$, so that $\operatorname{sp}(\bar{A})$ is closed in $E$ by $[(1) \Rightarrow(8)]$. Thus $s p(A)=s p(\bar{A}) \cap F$ is closed in $F$, and $F$ is dual locally complete by $[(8) \Rightarrow(1)]$.

Valdivia's proof only uses [(1) $\Rightarrow(8)]$, his Theorem 8 in [18]. His book ([19], p.47) presents the Saxon-Levin-Valdivia Theorem via condition (8), generally following the Saxon-Levin dichotomy which requires a separate closed subspace argument. Use of condition (7) permits a very quick, seamless proof:

Theorem 3.2 (Saxon-Levin-Valdivia). Every countable-codimensional subspace $F$ of a barrelled space $E$ is barrelled.

Proof. Let $B$ be a barrel in $F$ with closure $A$ in $E$. Let $H$ be an algebraic complement of $s p(A)$ in $E$. Theorem 2.10(7) implies $A+H$ is a barrel, hence a $0-$ neighborhood in $E$, and thus so is $B=(A+H) \cap F$ in $F$.

Suppose that: $u_{\iota}$ is a continuous linear map from a dual locally complete space $E_{\iota}$ into a space $E ;\left\{A_{n}\right\}_{n}$ is an absorbing sequence in $E$; and $f \in E^{*}$ with each $\left.f\right|_{A_{n}}$ continuous. Then $\left\{u_{\iota}^{-1}\left(A_{n}\right)\right\}_{n}$ is an absorbing sequence in $E_{\iota}$ and each $\left.\left(f \circ u_{\iota}\right)\right|_{u_{\iota}^{-1}\left(A_{n}\right)}$ is continuous, so that $f \circ u_{\iota} \in E_{\iota}^{\prime}$. Therefore [(1) $\left.\Leftrightarrow(3)\right]$ of Theorem 2.3 yields:

Theorem 3.3. Dual local completeness is preserved by inductive limits.

Corollary 3.4. Dual local completeness is preserved by:

(1) (Valdivia [19], 1.3.2.7) Hausdorff quotients;

(2) direct sums.

Theorem 3.5. The product $E=\prod_{\iota \in I} E_{\iota}$ of dual locally complete spaces $E_{\iota}(\iota \in I)$ is, itself, dual locally complete.

Proof. Let $A$ be an absolutely convex $\sigma\left(E^{\prime}, E\right)$-closed and bounded set in $E^{\prime}$. Boundedness implies a finite $J \subset I$ such that all members of $A$ vanish on $\prod_{\iota \in I \backslash J} E_{\iota}$ (canonically identified as a subspace of $E$ ). Thus if $B$ is the set of restrictions to $F=\prod_{\iota \in J} E_{\iota}$, we can identify $E_{A}^{\prime}$ with $F_{B}^{\prime}$, a Banach space, since the finite product $F$ is dual locally complete by Theorem 3.3 .

The three-space-problem for dual locally complete spaces has a negative solution that we plan to publish later. 


\section{REFERENCES}

1. M. De Wilde and C. Houet, On increasing sequences of absolutely convex sets in locally convex spaces, Math. Ann. 192(1971), 257-261. MR 44:5746

2. J. C. Ferrando, M. López Pellicer and L. M. Sánchez Ruiz, Metrizable Barrelled Spaces, Pitman RNMS 332, Longman, 1995. CMP 96:09

3. T. Husain, Two new classes of locally convex spaces, Math. Ann. 166(1966), 289-299. MR 35:3406

4. N. J. Kalton, Some forms of the closed graph theorem, Proc. Camb. Phil. Soc. 70(1971), 401-408. MR 46:634

5. G. Köthe, Topological Vector Spaces I, Springer-Verlag, 1969. MR 40:1750

6. M. Kunzinger, Barrelledness, Baire-like-, and (LF)-spaces, Pitman RNMS 298, Longman, 1993. MR 95e: 46004

7. P. Pérez Carreras and J. Bonet, Barrelled Locally Convex Spaces, Math. Studies 131, North Holland, 1987. MR 88j:46003

8. W. Roelcke, On the finest locally convex topology agreeing with a given topology on a sequence of absolutely convex sets, Math. Ann. 198(1974), 57-80. MR 48:2705

9. W. Ruess, A Grothendieck representation for the completion of cones of continuous seminorms, Math. Ann. 208(1974), 71-90. MR 50:14147

10. W. Ruess, Generalized inductive limit topologies and barrelledness properties, Pac. J. Math. 63, no. 2(1976), 499-516. MR 54:3348

11. W. Ruess, Closed graph theorems for generalized inductive limit topologies, Math. Proc. Camb. Phil. Soc. 82(1977), 67-83. MR 56:3612

12. S. Saxon, Basis Cone Base Theory, Doctoral Dissertation, Florida State University, Tallahassee, Fla., 1969.

13. S. Saxon, Nuclear and product spaces, Baire-like spaces, and the strongest locally convex topology, Math. Ann. 197(1972), 87-106. MR 46:4140

14. S. A. Saxon and M. D. Levin, Every countable-codimensional subspace of a barrelled space is a barrelled space, Abstract 660-44, Notices of the Amer. Math. Soc. 15(1968), 1020.

15. S. Saxon and M. Levin, Every countable-codimensional subspace of a barrelled space is barrelled, Proc. Amer. Math. Soc. 29(1971), 91-96. MR 43:6691

16. M. Valdivia, Absolutely convex sets in barrelled spaces, Ann. Inst. Fourier 21(1971), 3-13. MR 48:11968

17. M. Valdivia, Mackey convergence and the closed graph theorem, Arch. Math. 25(1974), 649656. MR 51:11052

18. M. Valdivia, On quasi-completeness and sequential completeness in locally convex spaces, J. Reine Angew. Math. 276(1975), 190-199. MR 52:8870

19. M. Valdivia, Topics in Locally Convex Spaces, Math. Studies 67, North Holland, 1982. MR 84i:46007

20. J. H. Webb, Sequential convergence in locally convex spaces, Proc. Camb. Phil. Soc. 64(1968), 341-364. MR 36:5652

21. J. H. Webb, Countable-codimensional subspaces of locally convex spaces, Proc. Edinb. Math. Soc. 18(1973), 167-172. MR 47:7370

22. A. Wilansky, Modern Methods in Topological Vector Spaces, McGraw-Hill, 1978. MR 81d:46001

Department of Mathematics, University of Florida, PO Box 118000, Gainesville, FLORIDA 32611-8000

E-mail address: saxon@math.ufl.edu

EUiti-Departamento de Matemática Aplicada, Universidad Politécnica de Valencia, E-46071 Valencia, Spain

E-mail address: lmsr@mat.upv.es 